\title{
Lived experiences of pregnancy among women with sickle cell disease receiving care at Mulago hospital: a qualitative study
}

\begin{abstract}
Background: Women with sickle cell disease in Mulago National Referral Hospital face challenges when they become pregnant and they receive the same care as all other high risk pregnant women who come to the hospital. This study explored the lived experiences of pregnancy among women with sickle cell disease receiving care at Mulago National Referral Hospital.
\end{abstract}

Methodology: This was a qualitative phenomenological study conducted on 15 participants who were women with sickle cell disease with the experience of pregnancy. In-depth audio recorded interviews were conducted to collect data from women who were pregnant at time of study or had ever been pregnant aged 16 to 38 years of age with sickle cell disease. Recorded data was transcribed and analyzed using content thematic approach.

Results: This study revealed that pregnant women with sickle cell disease faced both negative and positive health care experiences and individual lived experiences of pregnancy. The few positive individual lived experiences were joy of motherhood and giving birth to child free of sickle cell disease whereas the negative individual lived experiences reported were recurrent painful crises, pregnancy loss, premature delivery, stigma and discouragement, relationship discord and desertion by spouse. There were few positive healthcare experiences reported in this study which included: dedicated care and support from health workers, referral to specialist services, support from their male partners and the negative healthcare experiences reported were delay to get medical assistance, informal hospital charges, unsatisfactory care, and advocacy for caesarian section Vs normal delivery.

Conclusion: Pregnant women with sickle cell disease lived with great expectation and in fear of being further weakened by the disease. Their experiences were in general negative at both individual, social and health system levels.

Keywords: lived experiences, sickle cell disease, pregnancy
Volume 5 Issue 4 - 2019

\author{
Tumwesige Kenneth,' Namagembe Imelda, ${ }^{2}$ \\ Kayiga Herbert,' Munube Deogratias, ${ }^{2}$ \\ Rujumba Joseph' \\ 'Makerere University, College of Health Sciences, Uganda \\ 2 Mulago National Referral Hospital, Uganda
} Correspondence: Tumwesige Kenneth, Makerere university
Obstetrics and Gynecology, Uganda, Tel +256392942249, Email tkabwoolikenneth.tk@gmail.com

Received: June 20, 2019 | Published: July 12, 2019
Abbreviations: SCD: sickle cell disease; MNRH, Mulago national referral hospital; MUK, Makerere University; MUST, Mbarara University of science and technology.

\section{Background}

Sickle cell disease (SCD) is defined as an autosomal recessive hemoglobinopathy that includes SCD ( $\mathrm{HbSS}$ ) and various compound heterozygous genotypes (e.g. sickle cell carrier (HbSC) or sickle cell b-thalassemia disease (HbSb-thal) characterized by chronic hemolytic anemia and vaso-occlusive complications. ${ }^{1}$ Sickle cell disease is the commonest hereditary disorder and affects 30 million people worldwide ${ }^{2}$ and about $80 \%$ of SCD cases are believed to be in Sub-sahara Africa ${ }^{3}$ with 300,000 children born with the condition each year ${ }^{4}$ and in Uganda alone 20,000 babies per year are born with SCD. ${ }^{5}$ Therefore, it's against this background that we explored the lived experiences of pregnancy among patients with SCD in MNRH.

Pregnancy is a potentially serious condition among women living with SCD and can leave them even more fragile and insecure ${ }^{6}$.The fear of death and the death of their child is justified by the history of women who experience these complications. The women become aware of this fear from the reports of other women affected by the disease or from those who have had similar experiences in the family. ${ }^{7,8}$

Health care services and health care professionals are poorly prepared to care for these women, especially during pregnancy, which can contribute to increase the insecurity and fear they experience in this life stage. Access to quality prenatal care is often hampered for women suffering from SCD, either by physical and economic limitations to access services, or by the discrimination suffered because of their race, gender and low social class, in which these women find themselves. The difficulty of access to hospitals is one of the main factors responsible for maternal deaths. ${ }^{6}$

However, while many previous studies have explored how SCD affects pregnancy outcomes, ${ }^{9}$ few studies have explored what women with SCD go through during pregnancy. This presents a missed opportunity for understanding the experiences that these women go through during pregnancy and impedes our ability to assist them to enjoy the same health care services that other women without SCD enjoy. With this study we bridged this gap by exploring the lived experiences of pregnancy among women with SCD receiving care from MNRH. 


\section{Methods}

\section{Study design}

This was a qualitative phenomenological study that explored the lived experiences of pregnant women with SCD receiving care in MNRH in Kampala, Uganda. Data collection took place between October to December 2018. The choice of the qualitative approach was based on the fact that this approach provides the opportunity to document lived experiences of pregnancy among women with sickle cell disease through more in-depth and detailed narratives.

\section{Study setting}

The study was conducted in MNRH in Kampala, Uganda's Capital City. The selection of the study site was based on the fact that this is the national referral hospital for the country and handles all the high risk pregnant mothers and therefore would be able to get a big number of women with sickle cell disease that were pregnant at the time of study or had ever been pregnant. At the time of study, MNRH was under restructuring and the department of obstetrics and gynecology was in Kawempe Hospital and hematology clinic was in Kiruddu Hospital.

\section{Recruitment of participants}

Mulago National Referral Hospital records offices were searched to get patient files from out-patients unit, ward records books and antenatal books so as to identify women with SCD managed before and those who were receiving care from the hospital at that time. From the findings a total of 113 women with SCD in the reproductive age had received care from both Kawempe and Kiruddu Hospitals. 113 phone contacts were extracted from the hospital records and phone calls made to the participants inviting them to the clinics.

From Kawempe Hospital, department of Obstetrics and gynecology 46 possible participants' phone numbers were extracted. On first analysis 11 phone numbers had errors like missing figures and therefore these were eliminated. The PI (PI) started making phone calls for the remaining 35 phone numbers and phone calls were made at least three times at different times of the day if the number was not available the first time. The PI determined that 8 phone numbers were not available and 10 phone numbers belong to relatives who were no longer staying with the woman with SCD and so these were also excluded. Thus the PI remained with 17 phone numbers of the women with SCD that were available, when the number went through, the PI would introduce himself, thank the woman for taking the call, request if they were able to talk to him and if they agreed the PI would tell them his intention of calling. Then inquire if they had been pregnant before or were currently pregnant at that time. Out of 17 women with SCD 7 of them had not been pregnant and had received care for general gynecological complications from MNRH whereas 10 of the women had received care for obstetrical or pregnancy related complications.

Only 7 of these women with history of pregnancy accepted to be part of the study while 3 of them declined to participate in the study. Four women had interviews in the hospital and 3 at home. Antenatal and postnatal women with SCD admitted on the ward or attending antenatal care during the period of the study were recruited after they had stabilized or given appointments to return after discharge.

From Kiruddu Hospital, the hematology clinic record files were searched to look for women with SCD who were in the reproductive age and a total of 67 phone contacts of women with SCD were extracted and initial analysis by the PI revealed that 16 of the phone contacts had an errors like incomplete figures and 51 phone contacts were complete to the standard numbers and phone calls were made by the PI to book for appointments if the number went through. If the phone number wasn't available the first time, it was tried at least three times at different times of the day and 20 phone numbers were off and 12 were for relatives who reported that they were no longer staying with the woman with SCD, or the patient had passed on. Of the 19 women with SCD traced from Kiruddu hospital, 8 women consented to participate and so appointments were fixed by the PI with options of interviews done from hospital (5 women), home (1 woman) or at their place of work (2women), 10 women with SCD had not been pregnant before, 1 woman with SCD had been pregnant before but declined to participate. However the phone numbers that were off were tried on again after two weeks to see if the participant's phone was now available.

For interviews that were conducted in the hospital setting, we secured a consultation room in both Kawempe and Kiruddu Hospitals and requested the in-charges to give us the room for the interview without any other activity going on in that room. We always put a notice on the door and locked the doors to avoid interference from outside.

For interviews conducted at home, we agreed with the mothers to fix a date when she had enough help at home from someone who take away the baby for a few minutes and we sat in the house to avoid interference. For interviews at work place we scheduled interviews at hours that were not busy, one was a hotel manager while the second participant had a boutique shop in town and both recommended interviews are done early morning hours.

\section{Data collection}

A qualitative approach of semi-structured in-depth interviews was used to explore the lived experiences of pregnancy among women with SCD. The interview guide was in English and translated to Luganda to make it easy for participants to understand. Thus an appropriate language was used for each participant. Eight participants were called and accepted to come to the hospital and we requested those who couldn't come to the clinic to give us appointments and four participants preferred interviews done at their homes, two participants gave us appointments at their work places while only one participant was admitted in the hospital after delivery and she was interviewed on the day of discharge. This approach emphasis was to focus on capturing the participants' perspective, enlisting the participant as the expert knower, describing the participant's lived experience from their own perspective and searching for the meanings individuals give to their particular lived experiences. The responses of each participant were audio recorded using a recorder so that no information was lost.

\section{Data analysis}

This study used a descriptive phenomenology approach which favors thick description and close analysis of the lived experiences to understand how meaning is created through embodied perception. ${ }^{10}$ Data analysis followed the six steps of data analysis advanced by Colaizzi. ${ }^{11}$

Each interview was transcribed verbatim by a research assistant with experience in qualitative research to maintain contextual information. The individual identifiers were masked and transcripts 
were coded as P1-P15 to maintain anonymity. Each transcript was reviewed by the principal investigator alongside the audio-file for accuracy. The principal investigator then read through each transcript in order to become familiar with the collected data.

A code book was generated to give description of codes, significant and research relevant phrases were extracted from the transcripts by coding. This involved generating an initial list of concepts and themes by reading the data and creating as many relevant codes as possible and grouping the data accordingly.

The codes/ themes were sorted according to each participant using a coding framework and matrix to explore whether they have an indication of similar or disparate experiences. Each of the above steps was repeated for each transcript and organized to illustrate an aggregated meaning. The main themes that emerged from the above steps of analysis of the interviews were described and used to give an exhaustive description of the investigated phenomenon to understand the lived experience of women with SCD regarding pregnancy.

\section{Ethical consideration}

Permission to do the research was sought from School of Medicine Research Ethics Committee (SOMREC), Uganda National Council for Science and Technology, Department of Obstetrics and Gynecology and Hematology department of Mulago National Referral Hospital.

The study was individually explained to each participant and they were also encouraged to ask questions and seek clarification on anything related to the study. Informed consent was obtained from all study participants. Each study participant gave an informed consent and this obtained from the study participants before data was collection. Study participants under 18 years were considered as emancipated minors gave their own consent for participation in the study.

Confidentiality was maintained for all participants by ensuring that all the members of the research team understood what confidentiality meant and ensured that all the participants' information was only accessed by the study staff and the consent forms and transcripts were kept under lock and key. To further maintain confidentiality, the study participants' names were not used but rather study numbers were assigned to keep their identity anonymous.

\section{Results}

\section{Participant characteristics}

A total of 15 women with sickle cell disease participated in the study, of whom 12 had a history of pregnancy and 3 were pregnant at the time of the study. Seven of the study participants were from Kawempe hospital and 8 were from Kiruddu hospital. The age range of participants was 16-38, most of the women with sickle cell disease in this study were single and majority had attained secondary education. Majority of the participants had prior pregnancy experience and only a few were currently pregnant at the time of the study (Table 1).

\section{Lived experiences of pregnancy among women with SCD receiving care from $M N R H$}

The study explored lived experiences of pregnancy among women with SCD receiving care from MNRH and the findings are summarized under two major themes 1) Individual lived experiences and 2) health care service experiences as shown in Table 2.

Table I characteristic of women with sickle cell disease

\begin{tabular}{lll}
\hline Categories & Number & Percentage (\%) \\
\hline Age & 4 & $26.6(\%)$ \\
$15-25$ & 7 & $46.6(\%)$ \\
$26-35$ & 4 & $26.6(\%)$ \\
$36-45$ & & \\
Marital status & 8 & $53.3(\%)$ \\
Single & 7 & $46.6(\%)$ \\
Married & & \\
Education & 1 & $6.6(\%)$ \\
Primary & 12 & $80.0(\%)$ \\
Secondary & 2 & $13.3(\%)$ \\
Tertiary & & \\
Pregnancy status & 12 & $80.0(\%)$ \\
Prior pregnancy & 3 & $20.0(\%)$ \\
Currently pregnant & & \\
Place of interview & & $60.0(\%)$ \\
Hospital & 9 & $26.6(\%)$ \\
Home & & $13.3(\%)$ \\
Work place & & \\
\hline
\end{tabular}


Table 2 Lived experiences of pregnancy among women with SCD receiving care from MNRH

\begin{tabular}{lll}
\hline Sub themes & Theme & Phenomenon \\
\hline Positive Experience & Individual lived experiences & Lived experiences of pregnancy among women with SCD \\
Joy of motherhood & \\
Giving birth to a baby without SCD & \\
Negative Experiences & \\
Recurrent painful crises & \\
Pregnancy loss & \\
Premature delivery & \\
Stigma and discouragement \\
Relationship discord and desertion \\
by spouse \\
Positive Experiences \\
Dedicated care and support from \\
health workers \\
Referral to specialist services \\
Support from their male partners \\
Negative Experiences \\
Delay to get medical assistance \\
Informal hospital charges \\
Unsatisfactory care \\
Advocacy for caesarian section Vs \\
normal delivery
\end{tabular}

\section{Individual lived experiences}

Women with SCD in this study expressed both positive and negative individual lived experiences of pregnancy. A few of the study participants had positive lived experiences like Joy of motherhood and giving birth to a baby without sickle cell disease like the mother. Most of the women had negative lived experiences such as recurrent painful crises, pregnancy loss, premature delivery, stigma and discouragement, relationship discord and desertion by spouse.

\section{Positive experience}

\section{Joy of motherhood}

Only a few of the participants reported great desire to have a baby regardless of the advice to abort and were willing to risk their lives to become a mother as this would make them happy as in the excerpt below;"Okay there is a certain doctor who told me that the sickler's should not get pregnant because if you get pregnant, you can easily lose your life or the baby will die. So it is best we remove the baby now. Then I just told the doctor, ahh it's just a gift from God. So, if it goes well that's what God wants but if I die, that's what God wants but for me, I will have my baby." (pg 2 line 51-58) P1.

\section{Giving birth to a baby without sickle cell disease like the mother}

One Mother who had given birth to a child without sickle cell disease expressed happiness knowing that her child will not be affected by SCD as narrated below; "I remember I received like 3 units of blood, yeah but the good chance I got was the baby was not affected with the sickness." (pg 2 line 38-41) P6.

\section{Negative experiences}

Most of the mothers in our study narrated the dark side of their individual experiences of pregnancy as described in the following subsections.

\section{Recurrent painful crises}

Most of the mothers narrated how they had experienced painful crises during the pregnancy, having severe body pains and struggling with their health status often resulting into frequent hospitalization and blood transfusions conditions uncommon to their non- sickle cell disease counterparts as the mothers explained; "I went through a lot painful experiences; I could be feeling dizzy, joint pains...I even got an attack and could get severe headaches every now and then. It is during this time that I went to hospital and was admitted." (Page 1 Line 12-14) P10.

For most mothers keeping a stable health status was an uphill task, especially the mother who had been pregnant more than once noted that the complications in SCD such as pain, fever, blood transfusions increased during pregnancy as explained below by one of them."Again while pregnant I got a fever, and when I went to the clinic the doctor reported that I was anemic. I was transfused with 5 bottles of blood and 4 of drip water. I had fear for my life because I had heard from people that blood from [mentions name of hospital] is infected; with 
this wrong information I ended up running out of hospital." (Pg1 Line 11-14) P8.

\section{Pregnancy loss}

Three of the participants had experienced a miscarriage or intrauterine fetal death a process they described as difficult and painful as one was quoted in the excerpt below; "Okay my condition was not good so they gave me some injections and suddenly they told me that my baby had died in the stomach and yet it was okay from before because I went for scanning on Monday then again on Friday. They told me to go back again for scanning. Then when I went there, they told me that the baby died and yet the baby had been okay. It was painful" (pg 1 line 12-18) P1.

\section{Premature delivery}

One of the mothers narrated that carrying the child to full term was a challenge as she ended up having to give birth earlier than expected and caesarian delivery was done to save the baby as well as her life and the fear of death was evident for mothers in the study."They said this child is about to get delivered before it's mature but another doctor said let us first give her this medicine in the meantime so that the lungs can grow. So they injected me with medicine. That medicine was very painful but I didn't care about its pain. I just prayed to God for one thing that he may deliver me and my child. And I stayed in Mulago for a week because she was a premature." pg 2 line 50-53) P3.

\section{Stigma and discouragement}

A part from the community beliefs and expectation that women with SCD can hardly conceive and bare children, pregnant mothers also shared experiences of being stigmatized; receiving discouraging and negative comments from those around them. This created feelings of being alone and not cared for as one woman mention;"In most cases, the medical people blame you. You a sickler, why did you go for it? You knew, you think you can carry this pregnancy. You can't. You knew you were a sickler, why did you engage in sex to get pregnant?" (pg 9 line 285-287) P4.

Another woman carrying her first pregnancy narrated how she was being criticized and discouraged by health workers for being pregnant as sickler, she reported as quoted below; "When I went to the hospital for the first time, the doctor that attended to me told me not to get pregnant again and she went on to tell me I shouldn't have gotten pregnant because sicklers don't give birth and die at the time of delivery." (Page 4 Line 75-77) P12.

The stigma, discouragement and criticism received by pregnant mothers often led to anxiety and depression as one of the mothers carrying her second pregnancy expressed in the excerpt below;

"When my first born was 4yrs I conceived again because I could get different advice from different people not to at least stop at one child; while pregnant with this child, I became ill because I was depressed... There reached a time when I could be depressed that I will not make it. And on one occasion while I was pregnant I remember I lost my conscious and on gaining it after 3days I realized they had operated me and removed the baby after getting an attack that almost took my life." (Pg 1 Line 9-10) P8.

\section{Relationship discord and desertion by spouses}

Most mothers with SCD were single mothers as they reported being disserted by their male partners. It's should be noted that a positive relationship between pregnant women and their partners is vital as being deserted by their partners put a strain on their well being as one of the participants explained; "Now me, the one who said he would take responsibility when he knew that I was pregnant, he took off. So when he took off, family members were suggesting that I imprison him. But I refused. The man ran away completely..... When I tried to contact the family, for them they were saying ah ah we can't manage you. So I was left like that. So I carried the pregnancy up to, it was like even up to term." (pg 2 line 18-35) P4.

Some male partners after realizing the health condition of their partners fail to take their roles as fathers and this resulted in separation as one of the mothers narrated; "The biggest problem is with men, after impregnating you they deny you knowing you are a sickler... There is a friend of mine previously who consulted him [participant's partner]asking why he left and he was telling him that I am sick of HIV because of the sickle cell crises I was getting so throughout the pregnancy, the 9 months, he refused to give care. He was just quarrelling so I decided to give up with him because even the baby had died.” (pg 2 line 40-42) P9.

A few of the mothers noted that they had experienced desertion even from family members who at such times would have been a great source of support especially for those who had been abandoned by their partners as one of them is quoted below; "For even my family members disappeared. The bad thing is my mother and dad; they died a long time ago and my sisters also gave up on me..." (pg 2 line 4346) P4.

Abandonment left these women lonely and without anyone to care for hem especially where admission was involved as one of the women explained; "I was very okay. But this one came due to stress. So I was admitted. I was there alone. I was left alone.... After he left I felt lonely and all alone. It was not a nice experience. That is the first thing I told you. I had no one to take care of me.” (pg 4 line 151-154) P4.

\section{Health care experiences}

While participants recognized the importance of access to health care, there were several concerns that related to their experience with the facilities and the healthcare providers. A few were positive experiences divided into subthemes which included: dedicated care and support from health workers, referral to specialist services and support from their male partners while the majority had negative experiences with the health care they received summarized into subthemes including: delay to get medical assistance, unsatisfactory care and inappropriate health care systems

\section{Positive experiences}

\section{Dedicated care and support from health workers}

Empathy, humanity, understanding and respect were qualities of health care providers, including doctors and nurses that a few of the mothers appreciated and credited as in the narrative below; "Those people, they always attended to us in time. The results would come back on time, the feedback on how to solve the proble. They made sure that the baby inside is very fine, it was their routine to do that whenever we visited. And at least, they would look out for us." (pg 5-6 line 163-165) P7.

\section{Referral to specialist services}

Most of the mothers acknowledged that they were a special 
population from other women and required special care and attention. Thus attending a health facility where they were considered priority, having personnel offering a service and doing everything in their power to see that the mother is well created a favorable experience. However few of the mothers reported that they got linkage to specialist care which they received at MNRH as seen in the narratives below; "So when I went to the hospital, there is also our clinic, a sickle cell clinic. There is a doctor. He connected me to a specialist that I went to see. When I went, he told me some things and I did them".... (pg 3 line 78-81) P2. "And the doctor told me you should go to Mulago and get this person but I didn't know that [mentions specialist's name] was there and I received specialized care. So, it was just God. It was just God.” (pg 7 line 209-211) P2.

\section{Support from their male partners}

The other important positive health care experience that was revealed was having a supportive partner. This was important because the male partners would provide care and love which is key in the wellbeing of the pregnant mother in the hospital as one mothers narrated in the excerpt below;

"They tell us that we can't but me I have seen that if you have a good support, and when you are getting support, you are cared for, then you can carry the pregnancy up to term, that one, it is only care and love. When I was weak, he was there for me.” (pg 3 line 63-71) P4.

\section{Negative experiences}

\section{Delay to get medical assistance}

Most mothers had great delays in receiving medical assistance when they arrived at the health facility however these delays weren't unique for the mothers with SCD. For some delays in care resulted in worsening of the pain crises as expressed in the excerpts below. "In the morning, the doctor told me we have to have an operation and get the baby out. There was a doctor who came to check on me and told the nurse, take this one to the theatre so she can be operated upon at $2 \mathrm{pm}$.So I was there, waiting, knowing that at $2 \mathrm{pm}$, I am going to theatre but I spent two solid days and was not taken yet the doctor had even already signed the forms. Until at night, I got an attack. I am pregnant; I started getting pain in the stomach. Remember, the time had elapsed I was in so much pain....” (pg 2 line 56-70).

Delayed medical assistance was not only for in-patient mothers but similar experiences were witnessed by out-patient mothers who often became exhausted and frustrated as one participant expressed below. "You spend a long time waiting at the hospital... You come and sit for long, waiting. Sometimes you even go back without seeing someone... Sometimes you find the doctors tired. You find someone and he's not in the moods, he's not in the moods at all. And he is like "Ah you people, we are tired. Come back next time."(pg 7 line 214225) P2.

\section{Informal hospital charges}

Some of the delays in receiving health care related to inability to pay informal charges. One mother also noted that they were discriminated against on the basis of inability to pay the informal charges as she narrates below;

They didn't treat me well, even worse than the last time. We gave them the money. The one you give the money does not work at night.
There was a lot of money spent. We had to plead with them. Then when you give him 10,000shs and the other person has given him 20,000 shs so the one who gave him 10,000 shs is left and he goes with the one that gave him a lot more.

The notion 'No money, No care' seemed to resonate with some of the mothers who could not afford to offer some money and 'oil the health worker's hands ' [bribe] to the health worker in order to receive care and or receive care urgently even in circumstances of life and death."There was a friend who was also brought. She has SCD and had twins. As for her, she got pressure and even her children died but they didn't care. She was a poor woman.... the doctors would bypass her just like that until the girl got pressure. But if they had rushed her, they [the twins] would not have died. That is when I started getting worried. The things we go through are terrible. If you don't have money, you cannot give birth.”(pg 3 line 84-89) P13.

\section{Unsatisfactory care}

Despite waiting for long to see a health worker and being faithful to return to the clinic on their scheduled hospital visit days, the mothers relented that some of their visits were less informative and were not handled well by the health workers as most of the mothers narrated below; at the end of the day did not value their coming to the health facility or seeking medical assistance. "But for the pregnancy, I used to come here, on the day they have told you come back. You come, they check on you, but I don't know whether they used to estimate. They used to test the pressure, and they are telling me it is normal, it is normal but you are not well, till when I saw that it is too much and I said that let me go and try out at sickle cell clinic. Maybe I will get help." (pg 7 line 203-207) P2.

Two of the mothers narrated that many times during their hospital visits, they were attended to by a doctor who did not know much about SCD or did not know how to handle a pregnant woman with SCD. The mothers expressed the concern of limited knowledge by the healthcare workers more so handling pregnancy situations among women with SCD as reported in excerpt below; "It is just that when I get an attack; the problem is the other people don't really know much about SCD worse still when you are pregnant because I have been in so many health facilities, I have similar experience. You can reach the facility and you are the one to give them details that, "when am like this, they do this and that and treat me in this way' laughs." (Pg 3 Line 78-78) P13.

As a result of being less experienced in handling SCD patients, most mothers shared that many health care workers gave less care and attention to them despite their vulnerability. The mothers narrated that in some cases, the health workers were afraid or scared to handle/ care for a mother with such condition.“...even recently, they took me when I was heavy and in crisis, but reaching the emergency section at [mentions name of regional referral hospital], the nurses and doctors were just looking at me. No one even came near my bed. No one, not even one. Of course, the medical people we meet many times they don't understand sickle cell and yet they are the medical people who should know the condition but they don't understand." (pg 7 line 239240) P4.

Certainly, as a result of receiving sub-optimal care and attention, seeking antenatal care and support became unpopular to most mothers who never perceived the need for ANC if they had no complications. "People don't seem to care when you go. In the early months I had no complications whatsoever. My very first antenatal visit was at 
7months. I was forced to go because I felt heaviness and I had started feeling unwell. If it wasn't for the pain, I would not have gone." (Page 1 Line 10-11) P10.

\section{Advocacy for caesarian section Vs normal delivery}

To some mothers, the feeling that the health workers were less experienced to handle mothers with SCD is the reason they exhibited less care and attention. They believed that the health workers wanted to simplify their lives when handling pregnant women with SCD in labor by resorting/ advocating for $\mathrm{C}$-section against normal delivery.

"I went back for admission. My doctor promised me to take me for caesarean section with no explanation. So I was on the ward and I told the doctor that now it is full term and I have not heard any movement. But I had moved up to term so I could push.” (pg 2 line 35-37) P4.

\section{Discussion}

This phenomenological study explored the lived experiences of pregnancy among women with sickle cell disease. Most women had negative experiences at individual and health system levels. At individual level women experienced recurrent painful crises, pregnancy losses, premature delivery, stigma and discouragement, relationship discord and desertion by spouses which created anxiety and fear among pregnant women with sickle cell disease.

At health system level, the negative experiences women encountered were delay to get medical assistance, informal hospital charges, unsatisfactory care and advocacy for caesarian section against vaginal delivery. Taken together, the negative experiences women with sickle cell disease encountered contradicted their expectations and desire for motherhood.

The few positive experiences that came out strongly were the joy of motherhood and giving birth to baby free from sickle cell disease. At health system level, some women had been attended to by dedicated health care workers; some were referred to specialist services and others cherished the support from their male partners. These made their experience of pregnancy enjoyable despite the challenges of their condition.

The dominance of the negative experiences among pregnant women with SCD in this study can be explained by inadequate support at family, community and health system levels. The negative experiences were worsened by the wide spread belief that women with sickle cell disease shouldn't become pregnant because of the feared maternal and fetal complications. As a result women in this study lived in constant fear and had moments of depression. Our findings are similar to those of a study done in Brazil where pregnant women with SCD thus experienced sadness, depression and think of interrupting the pregnancy. ${ }^{6}$

The few women who described positive experiences drew on joy of motherhood, giving birth to baby free of sickle cell disease and special treatments they occasionally received from some health workers. Many of the women with SCD report a desire to realize the dream of their companions - paternity but also the desire for children so as to constitute a biological family, which emphasizes the strength of the marriage bond and gender differences. ${ }^{12}$

\section{Conclusions}

Pregnant women with SCD receiving care from MNRH had both individual lived experiences and health care experiences which were mostly negative experiences with a few positive experiences during pregnancy. Stigma and discouragement with relationship discord were viewed as the most negative experiences whereas the joy of motherhood and giving birth to child who was free from sickle cell disease were the main positive experiences for the mothers.

\section{Acknowledgments}

None.

\section{Conflicts of interests}

The authors declare that they have no competing interests.

\section{References}

1. Weatherall DJ. ABC of clinical haematology. The hereditary anaemias. BMJ. 1997;314(7079):492.

2. Hathaway AR. Sickle Cell Disease in Pregnancy. South Med J. 2016;109(9):554-556.

3. Rees DC, Williams TN, Gladwin MT, et al. Sickle-cell disease. The Lancet. 2010;376(9757):2018-2031.

4. Serjeant GR, Hambleton I, Thame M. Fecundity and pregnancy outcome in a cohort with sickle cell-haemoglobin $\mathrm{C}$ disease followed from birth. BJOG: An International Journal of Obstetrics \& Gynaecology. 2005;112(9):1308-1314.

5. Ndeezi G. Burden of sickle cell trait and disease in the Uganda Sickle Surveillance Study (US3): a cross-sectional study. The Lancet Global Health. 2016;4(3):e195-e200.

6. Xavier ASG. Perception of women suffering from sickle cell anemia regarding pregnancy: an exploratory study. 2013;12(4):790.

7. Nomura RMY. Maternal and perinatal outcomes in pregnancies complicated by sickle cell diseases. Revista Brasileira de Ginecologia e Obstetrícia. 2010;32(8):405-411.

8. da Cruz Santos AC. Feelings of women with sickle cell anemia with regard to reproductive experiences. Journal of Nursing UFPE on line. 2012;6(12):2934-2941.

9. Muganyizi PS, Kidanto H. Sickle cell disease in pregnancy: trend and pregnancy outcomes at a tertiary hospital in Tanzania. PloS one. 2013;8(2):e56541.

10. Creswell JW. Research Design. 2003.

11. Colaizzi PF. Psychological research as the phenomenologist views it. 1978.

12. Vargas EP, LdC Moás. Normative discourses on the desire to have children. Revista de saude publica. 2010;44(4):758-762. 